\title{
MAGNETIC EFFECTS ON TRIPLET EXCITON INTERACTIONS
}

\author{
R. E. MERRIFIELD \\ Central Research Department, E. I. du Pont de Nemours and Company, \\ Wilmington, Delaware 19898, USA
}

\begin{abstract}
The rates of many bimolecular reactions involving triplet excitons are magnetic field dependent at room temperature and for modest field strengths. The specific interactions discussed are the fusion of a pair of triplet excitons to yield either a single or triplet exciton, the interaction between a triplet exciton and a trapped triplet, the fission of a singlet exciton into a pair of triplets and the triplet excitonfree radical interaction. The magnetic field effects result from the influence of the field on the spin wavefunctions of the triplet exciton together with the existence of spin selection rules for the interactions. A simple theory of these effects is presented. Application of the theory to the experimental field dependence results yields new information about the mechanisms of these interactions.
\end{abstract}

\section{INTRODUCTION}

- Some of the most interesting properties of triplet excitons are consequences of the fact that they are triplets - that is, that they consist of three closely spaced levels whose energies and wavefunctions can be modified by the application of a magnetic field. Since triplet excitons in organic crystals typically have diffusion lengths of the order of 30 microns, they explore a large volume of the crystal during their lifetimes and have an opportunity to undergo a variety of interactions with any other objects which may be simultaneously present in the crystal ${ }^{1}$. Some of these interactions which have been experimentally demonstrated are summarized by the following 'chemistry':

$$
\begin{array}{lll}
T+T \rightarrow S_{1}+S_{0} & \text { Homofusion-Singlet channel } \\
T+T \rightarrow T+S_{0} & \text { Homofusion-Triplet channel } \\
T+t \rightarrow S_{0}+s_{1} & \text { Heterofusion } \\
S_{1}+S_{0} \rightarrow T+T & \text { Fission } \\
T+D \rightarrow S_{0}+D & \text { Triplet-Doublet interaction }
\end{array}
$$

where $S_{0}$ and $S_{1}$ represent molecules of the crystal in their ground and first excited singlet states, respectively, $T$ represents the lowest triplet state, and $D$ represents a doublet-state species (e.g. a free radical). The states designated 
by lower case letters represent analogous states localized at an impurity molecule in the crystal. It turns out that the rates of the reactions listed above are in many cases magnetic field dependent at room temperature and for modest field strengths - conditions under which spin polarization of the triplet exciton is completely negligible. It is the purpose of this review : first, to summarize the experimental findings on the magnetic field dependence of these reactions; second, to show that the field dependence can be understood quantitatively on the basis of a simple model of triplet exciton interactions; and finally, to show how application of the theory to the experimental results can provide a powerful tool for illuminating some rather subtle details of these interactions.

\section{SPIN STATES OF A TRIPLET EXCITON IN A FIELD}

Before turning to triplet-exciton interactions, it will be useful to review the effects of a magnetic field on the spin states of an individual triplet exciton since these spin states are intimately involved in determining the rates of the interaction processes.

It is well known that the spin states of a triplet exciton are described by the following spin Hamiltonian ${ }^{2}$ :

$$
\mathscr{H}=g \beta \mathbf{H} . \mathbf{S}+D S_{z}^{2}+E\left(S_{x}^{2}-S_{y}^{2}\right)
$$

where the first term describes the Zeeman interaction and the remaining terms represent the fine structure which results from the interaction between the magnetic dipoles of the two unpaired electrons which comprise the triplet state.

The fact that this spin Hamiltonian describes a triplet exciton rather than the triplet state of an isolated molecule appears only implicitly in the expression. The exciton Hamiltonian differs from the molecular one in two respects: first, the hyperfine structure term, which would be present in the molecular Hamiltonian as a result of the interaction between electron and nuclear spins, is averaged to zero by the rapid motion of the exciton; and second, the fine-structure tensor for the exciton is an average over the various inequivalent molecules in the crystal structure. This is also a consequence of the rapid motion of the exciton.

Turning next to the energies and wavefunctions of the triplet in a magnetic field, let us look first at the zero-field limit. One of the consequences of the presence of the fine-structure terms is to lift the degeneracy of the three triplet sublevels which would otherwise exist at zero field. The zero-field eigenstates are $^{3}$ :

$$
\begin{aligned}
& |x\rangle=2^{-\frac{1}{2}}(|-1\rangle-|+1\rangle) \\
& |y\rangle=i 2^{-\frac{1}{2}}(|-1\rangle+|+1\rangle) \\
& |z\rangle=|0\rangle
\end{aligned}
$$

whose energies are $D-E, D+E$, and 0 , respectively. The spin states on the right-hand side are quantized with respect to the $z$-axis of the fine-structure tensor. 
Turning next to the high-field limit, i.e. where the Zeeman energy is large compared to the fine structure splitting, the fine-structure terms can be treated as a first-order perturbation on the Zeeman levels $|0\rangle,|+1\rangle$ and $|-1\rangle$, which are quantized with respect to the external field. The result is that the energy levels are anisotropic - that is, they depend on the direction of the magnetic field with respect to the principal axes of the fine structure tensor. This is illustrated in Figure 1. Explicitly, the first-order energies are ${ }^{4}$ :

$$
\begin{aligned}
& E_{0}=(D-E) \cos ^{2} \alpha+(D+E) \cos ^{2} \beta \\
& E_{ \pm 1}= \pm g \beta H+D-\frac{1}{2}(D-E) \cos ^{2} \alpha-\frac{1}{2}(D+E) \cos ^{2} \beta
\end{aligned}
$$

where $\alpha$ and $\beta$ are the angles made by the field with the $x$ and $y$ principal axes, respectively, of the fine-structure tensor. However, to first order the spin wavefunctions follow the magnetic field and are not affected by the fine structure terms.
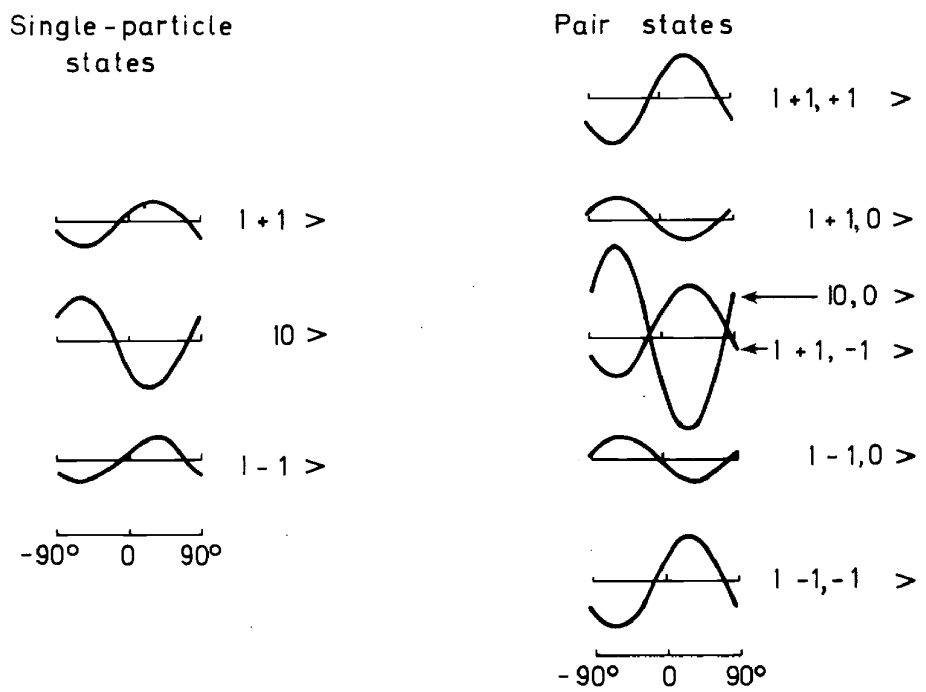

Figure 1. Anisotropy of energies in the high-field limit of the magnetic sublevels of a single triplet exciton and a pair of triplets in anthracene. The magnetic field lies in the $a c$ plane of the crystal and the indicated angles are with respect to the $a$ axis. The energy scale is arbitrary. (Reproduced by kind permission of the American Chemical Society. Accounts of Chemical

Research, 1, 129 (1968))

At intermediate fields where the fine structure and Zeeman terms are comparable, both the energy levels and wavefunctions of the triplet are complicated and rapidly varying functions of both the magnitude and direction of the applied field.

\section{TRIPLET EXCITON INTERACTIONS}

\section{A. Homofusion}

In many ways the most interesting interaction of a triplet exciton is the interaction with another identical exciton. The possible outcomes of an interaction between a pair of triplets are indicated in Figure 2 in which it is 

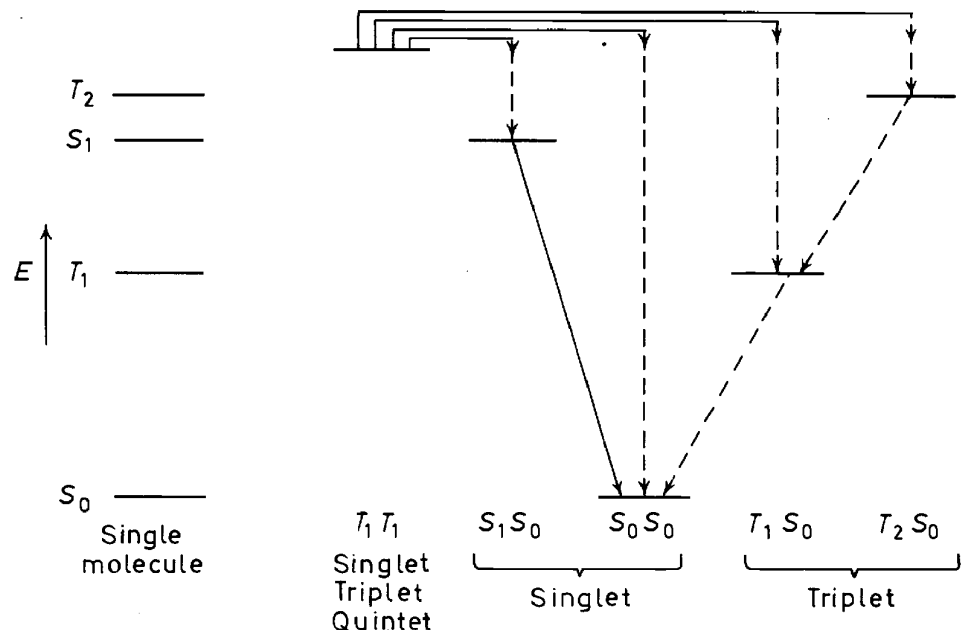

Figure 2. Electronic energy levels of a single molecule (far left) and of a pair of molecules showing the possible outcomes of the interaction of a pair of molecules in their triplet states. All of the indicated transitions are radiationless except that from $S_{1} S_{0}$ to $S_{0} S_{0}$, which is responsible for delayed fluorescence

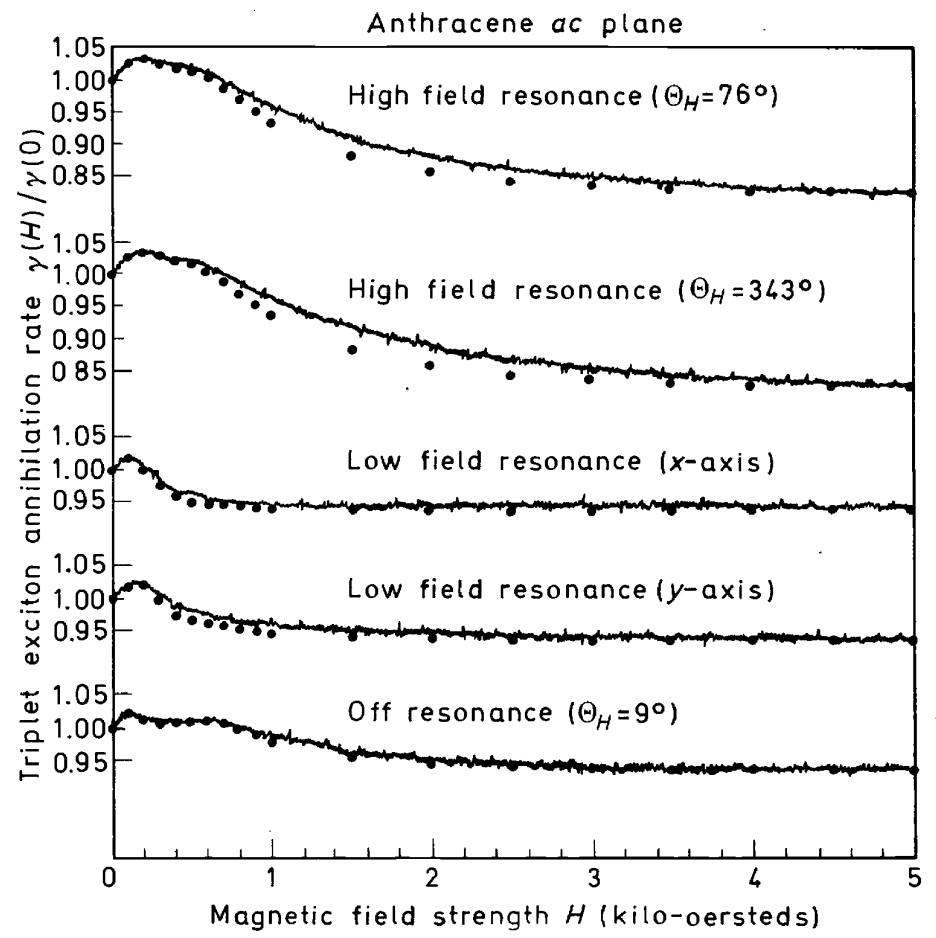

Figure 3. Experimental measurements and theoretical calculations (dots) of the field dependence of the singlet-channel fusion rate, $\gamma_{S}$, for several representative directions $\Theta_{H}$ in the ac plane of anthracene. The $x$ and $y$ axes of the dipolar tensor are at $\Theta_{H}=29.5^{\circ}$ and $299.5^{\circ}$, respectively.

(Reproduced by kind permission of the Physical Review. Phys. Rev. B1, 896 (1970).) 


\section{MAGNETIC EFFECTS ON TRIPLET EXCITON INTERACTIONS}

seen that there are several electronic energy levels of the pair which lie below the $2 T$ state, one of which, $S_{1}+S_{0}$, results in the subsequent emission of luminescence (delayed fluorescence). It is an experimental fact that delayed fluorescence resulting from the homofusion of triplet excitons is magnetic field dependent $t^{4,5}$. Typical experimental results for an anthracene crystal are shown in Figures 3 and 4.

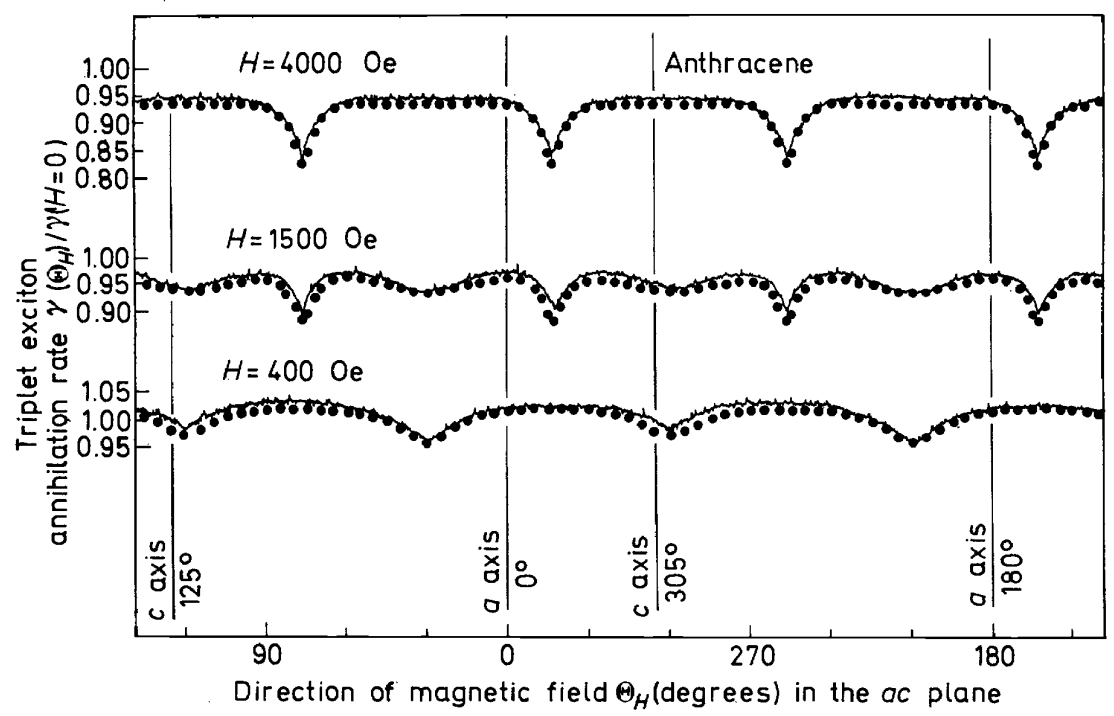

Figure 4. Experimental measurements and theoretical calculations (dots) of the anisotropy of the singlet-channel fusion rate, $\gamma_{s}$, in the $a c$ plane of anthracene for three magnetic field strengths. The high-field resonances are evident at $4000 \mathrm{Oe}$ and the low-field resonances at $400 \mathrm{Oe}$. At 1500 Oe the two sets of resonances coexist. The low-field resonance directions coincide with the $x$ and $y$ principal axes of the fine-structure tensor. (Reproduced by kind permission of the Physical Review. Phys. Rev. B1, 896 (1970))

In order to describe the interaction of a pair of triplets we require the spin Hamiltonian of a pair. The simplest assumption is that it is simply the sum of two single-particle Hamiltonians, i.e.

$$
\mathscr{H}=g \beta \mathbf{H} .\left(\mathbf{S}_{1}+\mathbf{S}_{2}\right)+D\left(S_{z_{1}}^{2}+S_{z_{2}}^{2}\right)+E\left(S_{x_{1}}^{2}+S_{x_{2}}^{2}-S_{y_{1}}^{2}-S_{y_{2}}^{2}\right)
$$

It could be argued that this Hamiltonian is obviously incomplete in that it contains no terms describing the interaction between the two triplets, and in fact such an interaction certainly must exist in order for the fusion process to take place. However, it turns out that this interaction is negligible with respect to its influence on the spin part of the pair wavefunction and the consequent field dependence of the fusion rate. Whether or not such an interaction is included, one of the most important properties of the pair Hamiltonian is the fact that it is invariant under interchange of the two excitons. This has the important consequence that the eigenstates can be classified into those which are even and odd under the interchange operation.

At zero field the pair eigenstates are those shown in Figure 5. It is seen that the interchange symmetry leads to a degeneracy in pair states such as $|x y\rangle$ 


\section{R. E. MERRIFIELD}

where the two excitons have different single particle states. None of the zero-field pair states corresponds to a definite spin multiplicity. This can be seen by examining the eigenstates of total spin expressed in terms of the zero-field pair wavefunctions. These are :

$$
\begin{array}{ll}
\text { Singlet: } & |S\rangle=3^{-\frac{1}{2}}(|x x\rangle+|y y\rangle+|z z\rangle) \\
\text { Triplets: } & \left|T_{x}\right\rangle=2^{-\frac{1}{2}}(|y z\rangle-|z y\rangle) \\
\text { Quintets: } & \left|Q_{a}\right\rangle=2^{-\frac{1}{2}}(|x x\rangle-|y y\rangle) \\
& \left|Q_{b}\right\rangle=6^{-\frac{1}{2}}(|x x\rangle+|y y\rangle-2|z z\rangle) \\
& \left|Q_{x}\right\rangle=2^{-\frac{1}{2}}(|y z\rangle+|z y\rangle)
\end{array}
$$

where the states $\left|T_{y}\right\rangle,\left|T_{z}\right\rangle$ and $\left|Q_{y}\right\rangle,\left|Q_{z}\right\rangle$ are obtained from $\left|T_{x}\right\rangle$ and $\left|Q_{x}\right\rangle$ by cyclic permutation of $x, y$ and $z$. Note that the singlet and quintet states are even under interchange of the two triplets, while the triplet states are odd. This is a general result regardless of the value of the magnetic field.

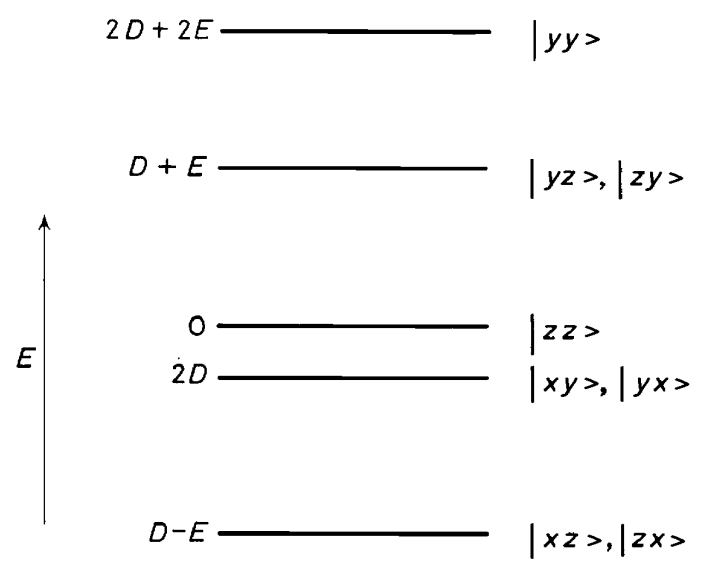

$2 D-2 E \longrightarrow \mid x x>$

Figure 5. Zero-field spin states and energy levels for a pair of triplet excitons. The states at $D+E, 2 D$ and $D-E$ are doubly degenerate

Turning next to the high-field limit, the anisotropies of the energies of the high-field pair states are shown in Figure 1. The pure spin states expressed in terms of these high-field, pair eigenstates are:

$$
\begin{aligned}
& |S\rangle=3^{-\frac{1}{2}}(|00\rangle-|+-\rangle-|-+\rangle) \\
& \left|T_{0}\right\rangle=2^{-\frac{1}{2}}(|+-\rangle-|-+\rangle) \\
& \left|Q_{0}\right\rangle=6^{-\frac{1}{2}}(2|00\rangle+|+-\rangle+|-+\rangle) \\
& \left|T_{ \pm_{1}}\right\rangle=2^{-\frac{1}{2}}(| \pm 0\rangle-|0 \pm\rangle) \\
& \left|Q_{ \pm 1}\right\rangle=2^{-\frac{1}{2}}(| \pm 0\rangle+|0 \pm\rangle) \\
& \left|Q_{ \pm 2}\right\rangle=| \pm \pm\rangle
\end{aligned}
$$




\section{MAGNETIC EFFECTS ON TRIPLET EXCITON INTERACTIONS}

Note that, as shown in Figure 1, for most field directions the two types of pair eigenstates which have a singlet component have different energies. However, there do exist particular field directions for which these states become degenerate so that at these level crossings a pure singlet eigenstate of the pair is possible ${ }^{4}$.

The next task is to relate the foregoing discussion of the pair spin states to the kinetics of the fusion process. This will be done in terms of the following simple kinetic scheme :

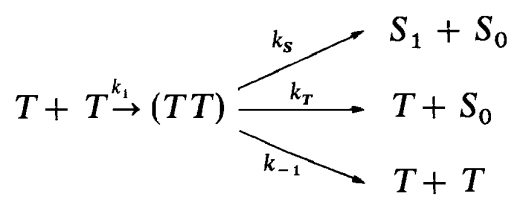

in which triplets come together to form pairs which can react via either a singlet or triplet channel or can scatter without reaction. It is assumed that the probability of fusion from a given one of the nine pair states is proportional to the spin amplitude for the appropriate channel. The kinetic equation for $P_{n}$, the steady state population of the $n^{\text {th }}$ pair states is ${ }^{6}$ :

$$
\frac{1}{9} k_{1}[T]^{2}=\left(k_{-1}+k_{S}\left|S_{n}\right|^{2}+k_{T}\left|\mathbf{T}_{n}\right|^{2}\right) P_{n}
$$

where

$$
\left|S_{n}\right|^{2}=\left|\left\langle S \mid \psi_{n}\right\rangle\right|^{2}
$$

and

$$
\left|\mathbf{T}_{n}\right|^{2}=\sum_{m=1}^{3}\left|\left\langle T_{m} \mid \psi_{n}\right\rangle\right|^{2}
$$

are the fractional singlet and triplet character, respectively, of the $n^{\text {th }}$ pair state, whose spin wavefunction is $\left|\psi_{n}\right\rangle$. Under steady-state conditions the total rates of fusion for the singlet and triplet channels become

$$
\begin{aligned}
& \gamma_{S}[T]^{2}=\sum_{n} k_{S}\left|S_{n}\right|^{2} P_{n}=\frac{k_{1}[T]^{2}}{9} \sum_{n} \frac{k_{S}\left|S_{n}\right|^{2}}{k_{-1}+k_{S}\left|S_{n}\right|^{2}+k_{T}\left|\mathbf{T}_{n}\right|^{2}} \\
& \gamma_{T}[T]^{2}=\sum_{n} k_{T}\left|\mathbf{T}_{n}\right|^{2} P_{n}=\frac{k_{1}[T]^{2}}{9} \sum_{n} \frac{k_{T}\left|\mathbf{T}_{n}\right|^{2}}{k_{-1}+k_{S}\left|S_{n}\right|^{2}+k_{T}\left|\mathbf{T}_{n}\right|^{2}}
\end{aligned}
$$

where $\gamma_{S}$ and $\gamma_{T}$ are the overall rate constants for the reactions

$$
\begin{gathered}
T+T \stackrel{\gamma_{s}}{\rightarrow} S_{1}+S_{0} \\
T+T \stackrel{\gamma_{T}}{\rightarrow} T+S_{0}
\end{gathered}
$$

This simple kinetic scheme is not really complete as a result of the degeneracies which can occur among the pair spin states. In the presence of a degeneracy the particular linear combinations of the degenerate pair of states which are to be used in calculating the spin amplitudes are ambiguous, and the value calculated for $\gamma_{S}$ and $\gamma_{T}$ will depend on what choice is made. A more 


\section{R. E. MERRIFIELD}

complete treatment of the dynamics of the interaction involving consideration of the density matrix of the pair spin states has been developed ${ }^{4}$ which resolves this ambiguity. Rather than giving the details of this treatment, we shall merely state the salient result, which is that in the presence of a degeneracy, that linear combination of the degenerate states is to be taken which comes closest to diagonalizing the total spin of the pair. Thus in the case of the degeneracy resulting from the interchange symmetry, even and odd combinations are to be used, the odd combination being in all cases a pure triplet. Similarly, at the high-field level-crossing pure singlet and quintet combinations are the appropriate pair states.

As a result of the considerations outlined in the preceding paragraph, it follows that the singlet and triplet channels for fusion are independent in the sense that no one pair state will simultaneously have both singlet and triplet character. Thus $\gamma_{T}$ is field independent (since $\left|\mathbf{T}_{n}\right|^{2}=1$ for a pure triplet, regardless of the triplet basis states ${ }^{7}$ ) and the expression for $\gamma_{s}$ becomes

$$
\gamma_{S}=\frac{k_{1}}{9} \sum_{n} \frac{k_{S}\left|S_{n}\right|^{2}}{k_{-1}+k_{S}\left|S_{n}\right|^{2}}
$$

The singlet amplitudes are constrained by the sum rule $\sum_{n}\left|S_{n}\right|^{2}=1$ and the value of $\gamma_{s}$ depends on the manner in which this unit of singlet character is distributed over the pair states. This can be seen by considering the simple situation in which $N$ of the pair states have equal singlet character, i.e. $\left|S_{n}\right|^{2}=1 / N$ for $N$ states and is zero for the rest. In this case

$$
\gamma_{S}(N)=\frac{k_{1}}{9} \times \frac{k_{S}}{k_{-1}+\left(k_{S} / N\right)}
$$

which is a monotonically increasing function of $N$. This illustrates the salient characteristic of equation (17), namely, that $\gamma_{s}$ increases as the singlet character becomes spread over more of the pair states and has its minimum value if one pair state is a pure singlet.

The magnetic field dependence of the fusion rate is thus described by equation (17), together with the field dependence of the pair spin wavefunctions and consequent field dependence of the $S_{n}$. The principal qualitative features of the magnetic field dependence of $\gamma_{s}$ shown in Figures 3 and 4 can now be accounted for. At zero field there are, from equation (10), three pair states which have singlet character, i.e. $|x \dot{x}\rangle,|y y\rangle$ and $|z z\rangle$. At high fields it is seen from equation (11) that there are in general two such states, i.e. $|00\rangle$ and $2^{-\frac{1}{2}}(|+-\rangle+|-+\rangle)$, so that $\gamma_{s}$ will be less than at zero field. At the particular field orientations for which these two states are degenerate, the pure singlet state becomes an eigenstate and $\gamma_{s}$ will decrease further (a level-crossing resonance). At intermediate fields the singlet character will be distributed over all of the even pair states and $\gamma_{s}$ will be larger than the zero-field value. This is illustrated in Figure 6 which shows how the magnetic field dependence of $\gamma_{S}$ is related to the field dependence of the energy levels and the distribution of singlet character over the six even states of the triplet pair. It is seen that the region of rapid variation of $\gamma_{S}$ with field occurs, as 


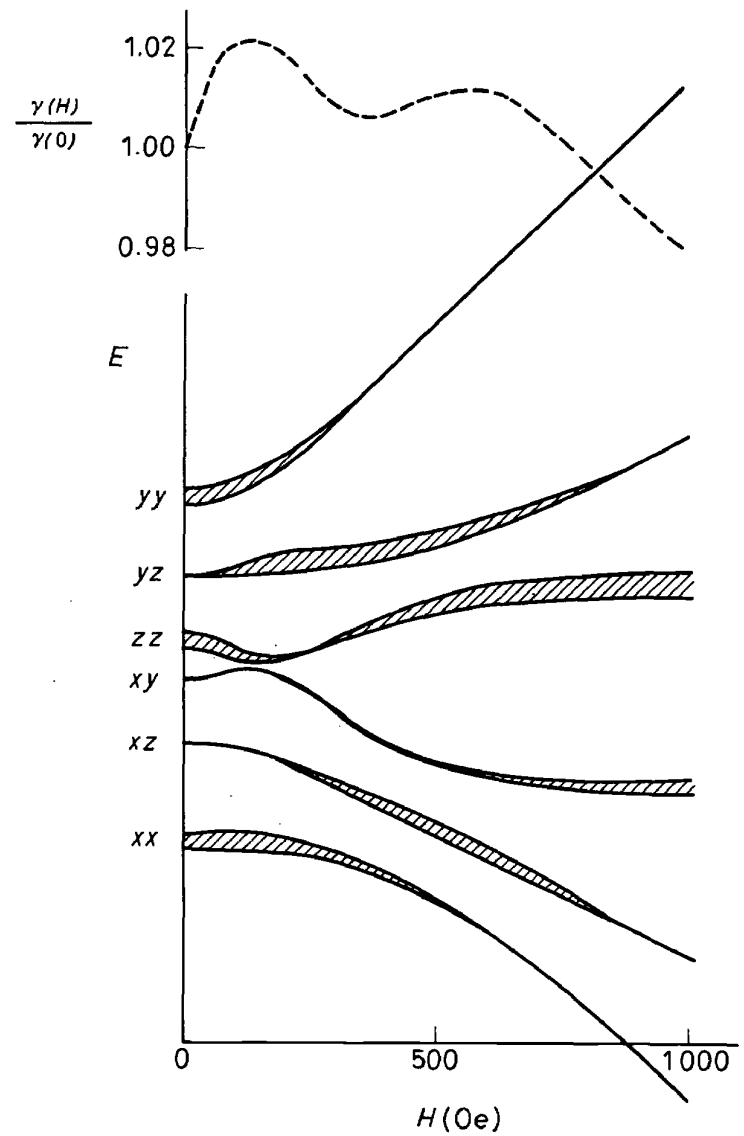

Figure 6. Typical calculated variation at low fields of the pair spin energy levels (lower solid curves) and singlet-channel fusion rate (upper dashed curve). The heights of the cross hatched areas are proportional to the fractional singlet characters of the pair states. The parameters employed in the calculation are $: D=-56 . \mathrm{Oe}, E=350 \mathrm{Oe}$ and $k_{s} / k_{-1}=0.4$

expected, in the region where the Zeeman energy and zero-field splitting are comparable.

The only case so far in which detailed experimental data exist and have been quantitatively compared with calculations based on this model is that of the anthracene crystal ${ }^{4}$. Typical experimental results along with the calculated points are shown in Figures 3 and 4 . The parameter values which give the best fit to the experimental data are the following:

$$
\begin{aligned}
k_{-1} & =(2.8 \pm 0.3) \times 10^{9} \mathrm{sec}^{-1} \\
k_{S} & =(1.1 \pm 0.1) \times 10^{9} \mathrm{sec}^{-1} \\
D & =-56 \pm 10 \mathrm{Oe} \\
E & =350 \pm 30 \mathrm{Oe}
\end{aligned}
$$




\section{R. E. MERRIFIELD}

For comparison, the values of $D$ and $E$ measured directly by ESR $^{8}$ are $-62 \pm 15$ Oe and $350 \pm 20$ Oe, respectively.

This does not yet yield a complete picture of the fusion process. Information is still lacking on the importance of the triplet channel as a consequence of its magnetic field independence and the fact that it does not lead to delayed fluorescence. However, the field independence of $\gamma_{T}$ can be exploited in a slightly modified experimental situation to give the desired information ${ }^{7}$. The basic idea is to examine the field dependence of delayed fluorescence under conditions of high exciton density in which the fusion channel becomes important in determining the steady-state exciton concentration so that one can examine the competition between the singlet and triplet channels. In the high-density limit where the bimolecular decay of triplets dominates the monomolecular decay, the steady-state triplet concentration, $[T]$, is determined by

$$
\alpha=\left(2 \gamma_{S}+\gamma_{T}\right)[T]^{2}
$$

where $\alpha$ is the rate of triplet generation and the coefficients of $\gamma_{S}$ and $\gamma_{T}$ reflect the fact that a singlet-channel fusion event removes twice as many triplets as a triplet-channel event. The delayed fluorescence intensity, $\phi$, is proportional to

$$
\gamma_{S}[T]^{2}=\frac{\gamma_{S}}{2 \gamma_{S}+\gamma_{T}} \alpha
$$

It can be seen that measurement of the field dependence of $\phi$ under these conditions will yield information on the relative magnitudes and field dependences of $\gamma_{S}$ and $\gamma_{T}$. Thus, if these two quantities should have the same field dependence, the delayed fluorescence would become field independent in the high-density limit. However, if, as expected theoretically, $\gamma_{T}$ is a constant, then the field dependence of $\phi$ will be reduced in amplitude from the low-density results and the amount of this reduction in amplitude will yield the ratio $\gamma_{T} / \gamma_{s}$. Groff, et al. ${ }^{7}$, have carried out such high-density experiments which confirm that $\gamma_{T}$ is indeed field independent and yield $\gamma_{T} / \gamma_{S}(0)=3.5 \pm 0.2$. Combining this result with the previous ones, we arrive at the following picture of the triplet fusion process:

$$
\begin{aligned}
& T+T \stackrel{k_{1}}{\rightarrow}(T T) \underset{k_{T}}{\stackrel{k_{-1}}{k_{s}}-T+T} \begin{array}{c}
T \\
\longrightarrow
\end{array} S_{1}+S_{0} \\
& k_{-1}=(2.8 \pm 0.3) \times 10^{9} \mathrm{sec}^{-1} \\
& k_{S}=(1.1 \pm 0.1) \times 10^{9} \mathrm{sec}^{-1} \\
& k_{T}=(1.7 \pm 0.2) \times 10^{9} \mathrm{sec}^{-1}
\end{aligned}
$$

From these values of the rate constants, together with equation (17), it it can be concluded that of the encounters of two triplet excitons 4 per cent lead to fusion via the singlet channel, 13 per cent to triplet-channel fusion and the remaining 83 per cent scatter without reaction. This example 
illustrates the power of the magnetic field experiments in illuminating many of the details of triplet-exciton interactions which are not accessible by any other means.

The homofusion of triplet excitons has been observed in many other organic crystals and in most cases exhibits a magnetic field dependence qualitatively similar to that seen in anthracene, although anthracene remains the only case in which really detailed results exist at present. One crystal in which qualitatively different behaviour is found is 9,10-diphenylanthracene. The field dependence of delayed fluorescence from this crystal is shown in Figure 7. The behaviour at low fields is similar to that of anthracene, but three additional peaks are found at 5.5, 11 and $22 \mathrm{kOe}$. The reason for the existence of this additional structure at high fields is not really understood,

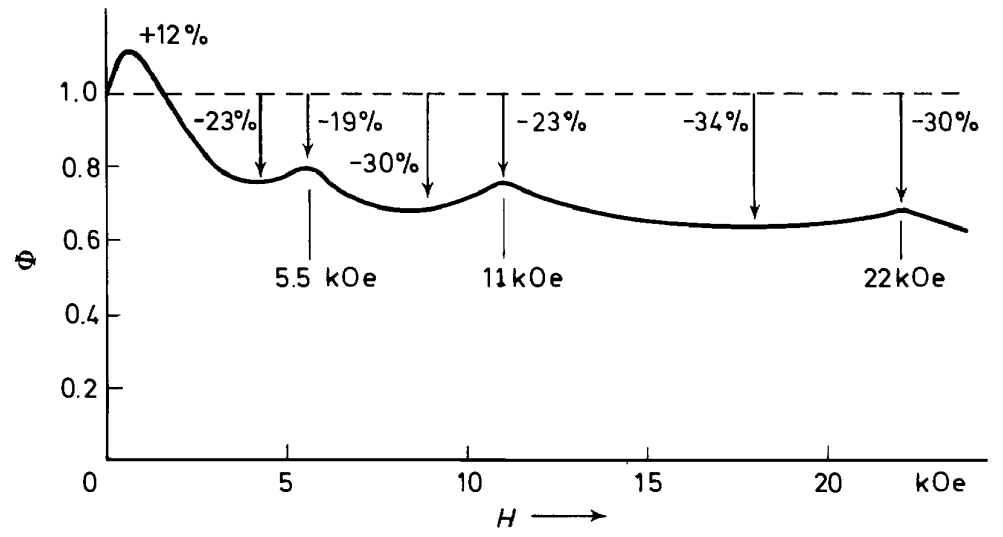

Figure 7. Magnetic field dependence of delayed fluorescence intensity from a 9,10-diphenylanthracene crystal of unknown orientation

but it can be partially accounted for on the assumption that for some unknown reason there exist two kinds of triplet excitons in the crystal which differ in energy by about $1 \mathrm{~cm}^{-1}$. The high-field energy levels in such a situation are shown in Figure 8. It is seen that the singlet-containing $\left(M_{S}=0\right)$ levels of the pair are crossed at three different fields (in the ratio of $1: 2: 4$ ) by levels which have no singlet character $\left(M_{S}= \pm 1, \pm 2\right)$. This is in contrast to the type of level crossing discussed earlier for anthracene in which two levels, both of which have singlet character, cross with a resulting decrease in fusion rate. This type of level crossing, i.e. a singlet and a pure nonsinglet, would be expected to lead to an increase in fusion rate as observed experimentally. Whether or not this picture bears any relation to the true explanation, it is clear that additional information about triplet excitons in this crystal is contained in the high-field structure.

Another type of triplet homofusion process is the reaction between triplet states of molecules in solution ${ }^{9}$. This process, too, is magnetic field dependent ${ }^{10.11}$ as illustrated in Figure 9 for delayed fluorescence resulting from fusion of triplet-state anthracene molecules in ethanol solution. The observed field dependence is accounted for on the basis of a model similar to that outlined above for crystals ${ }^{11}$. However, it is considerably more 


\section{R. E. MERRIFIELD}

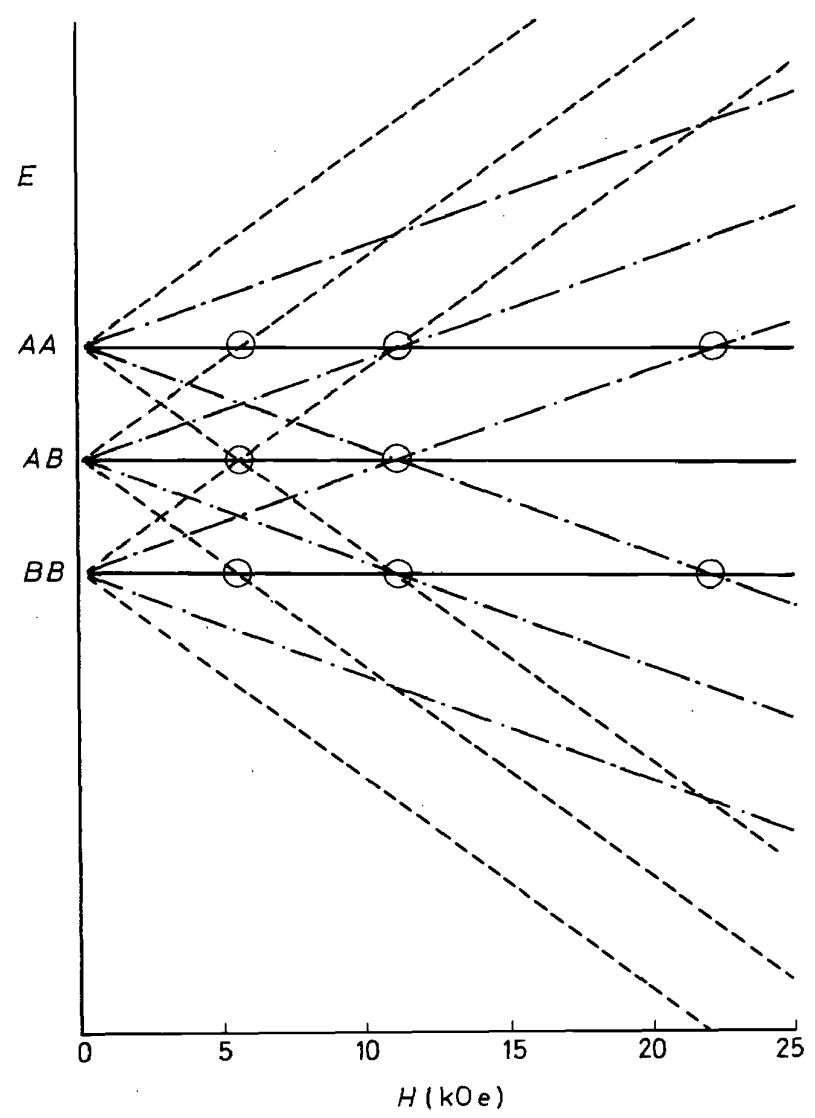

Figure 8. Hypothetical energy level scheme suggested by experimental results on 9,10-diphenylanthracene. There are two types of triplet excitons, denoted $A$ and $B$, giving rise to three types of pairs. The Zeeman levels are $M_{S}=0$ (solid lines), $M_{S}= \pm 1$ (dot-dash) and $M_{S}= \pm 2$ (dashes).

Level crossings involving the $M_{\mathrm{S}}=0$ levels are indicated by circles

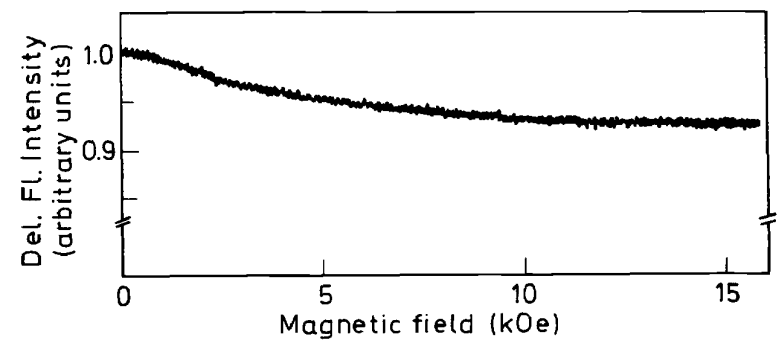

Figure 9. Dependence of delayed fluorescence intensity on magnetic field strength for a $10^{-4} \mathrm{M}$ solution of anthracene in ethanol at $25^{\circ} \mathrm{C}$. (Reproduced by kind permission of Academic Press, New York. Proceedings of the International Conference on Organic Scintillators and Liquid Scintillation Counting, San Francisco, July 1970) 
complicated in detail because of the necessity for averaging over all the possible molecular orientations which can occur in solution. Analysis of the experimental field dependence curve yields $k_{S} / k_{-1} \simeq 12$ for the solution experiment of Figure 9, compared to the value of 0.4 for this ratio in the anthracene crystal. Thus, in contrast to the situation in the crystal, triplet fusion in solution is diffusion limited.

\section{B. Heterofusion}

In the discussion of the preceding section it is clear that the symmetry of the pair spin Hamiltonian plays a dominant role in determining the magnetic field dependence of the homofusion rate constant. It is therefore of interest to examine the consequences of removing this symmetry. This can be done by looking at delayed fluorescence resulting from the fusion of a triplet exciton with a triplet state of an impurity molecule incorporated in the crystal ${ }^{12}$ (a localized exciton), i.e. heterofusion, the process of equation (3). Since the interchange symmetry is destroyed in this situation, the separation of the triplet pair states from the singlets and quintets is no longer obtained. One of the consequences of this broken symmetry is that we must now use the expression (14) rather than equation (17) for the fusion rate constant since now a given pair state may have both singlet and triplet character simultaneously. At zero field the following relation between singlet and triplet amplitudes can be shown to hold ${ }^{12}$ :

$$
\left|\mathbf{T}_{n}\right|^{2}=\frac{1}{2}-\frac{3}{2}\left|S_{n}\right|^{2}
$$

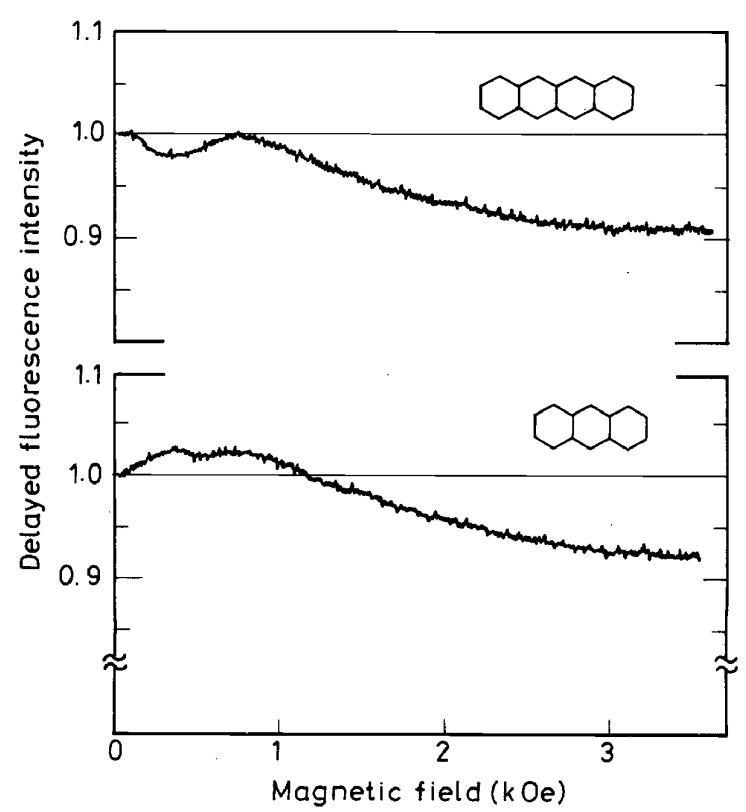

Figure 10. Field dependence of delayed emission from tetracene (heterofusion, top curve) and from anthracene (homofusion, bottom curve) in an anthracene crystal doped with $0.01 \mathrm{ppm}$ tetracene. The field is oriented along the $a$ axis. (Reproduced by kind permission of the Physical Review. Phys. Rev. Letters 25, 105 (1970)) 


\section{R. E. MERRIFIELD}

If this relation is used to eliminate the triplet amplitudes from equation (14), the result is

$$
\gamma_{S}=\frac{k_{1}}{9} \sum \frac{k_{S}\left|S_{n}\right|^{2}}{\left(k_{-1}+\frac{1}{2} k_{T}\right)+\left(k_{S}-\frac{3}{2} k_{T}\right)\left|S_{n}\right|^{2}}
$$

This has the same form as equation (17) for homofusion, but with the important difference that the coefficient of $\left|S_{n}\right|^{2}$ in the denominator need not be positive. If this coefficient is negative, i.e. if $k_{S}<\frac{3}{2} k_{T}$, then the dependence of $\gamma_{\boldsymbol{s}}$ on the way in which the singlet character is distributed over the pair states will be the inverse of that for homofusion. In particular, the initial field dependence would be a decrease in $\gamma_{s}$ rather than an increase.

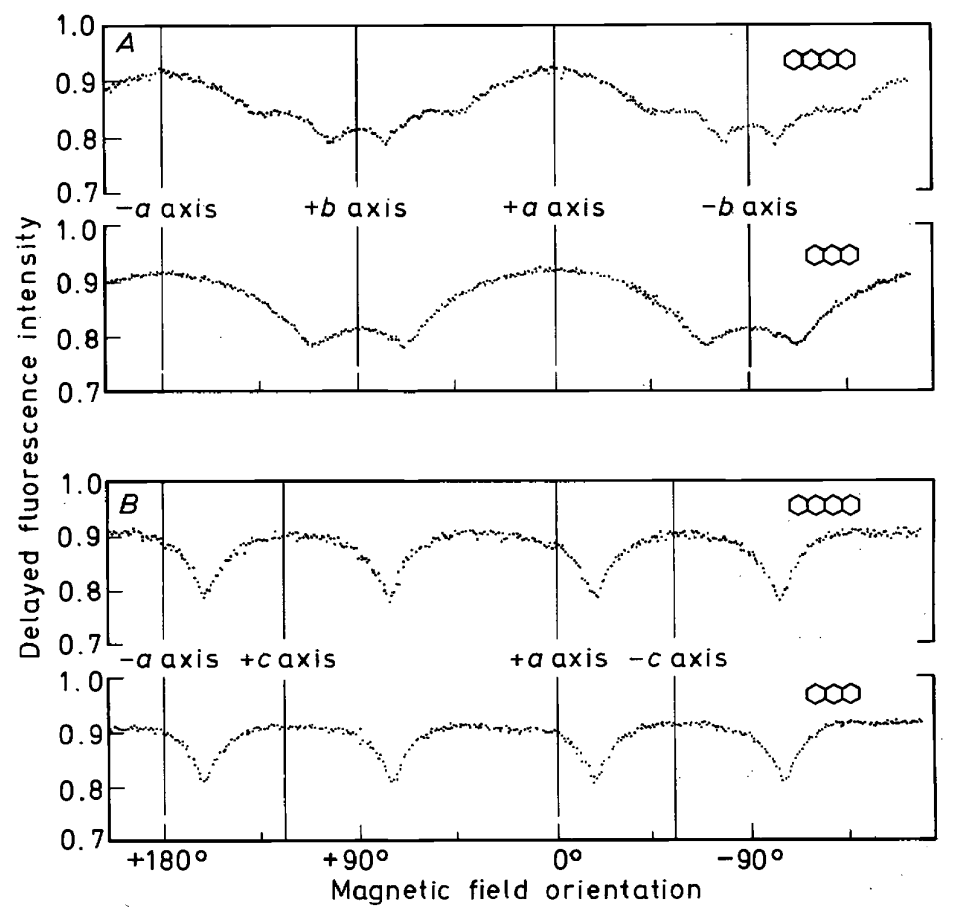

Figure 11. Dependence of tetracene and anthracene components of delayed fluorescence emission on the orientation of a $4 \mathrm{kOe}$ magnetic field in the $a b$ (top) and $a c$ (bottom) planes of an anthracene crystal doped with $0.01 \mathrm{ppm}$ tetracene. (Reproduced by kind permission of the Physical Review.

Phys. Rev. Letters 25, 105 (1970))

Figure 10 shows the field dependence of the heterofusion rate in a crystal of anthracene doped with $c a .0 .01 \mathrm{ppm}$ tetracene ${ }^{12}$. In this case the initial field dependence is indeed a decrease, thus confirming the qualitatively different nature of the heterofusion process. Following the initial decrease, at higher fields the field dependence resembles that for homofusion. This is presumably a result of the increasing importance of the (symmetric) Zeeman term in the pair spin Hamiltonian vis-à-vis the (unsymmetrical) fine-structure terms although explicit calculations of the theoretical field dependence do not 


\section{MAGNETIC EFFECTS ON TRIPLET EXCITON INTERACTIONS}

yet exist. The high-field anisotropy shown in Figure 11 shows another interesting qualitative difference from homofusion. The additional structure apparent in the figure is a result of the fact that heterofusion events involving a free exciton and triplets trapped on tetracene molecules at either of the two possible substitutional sites in the anthracene crystal structure ${ }^{13}$ can be

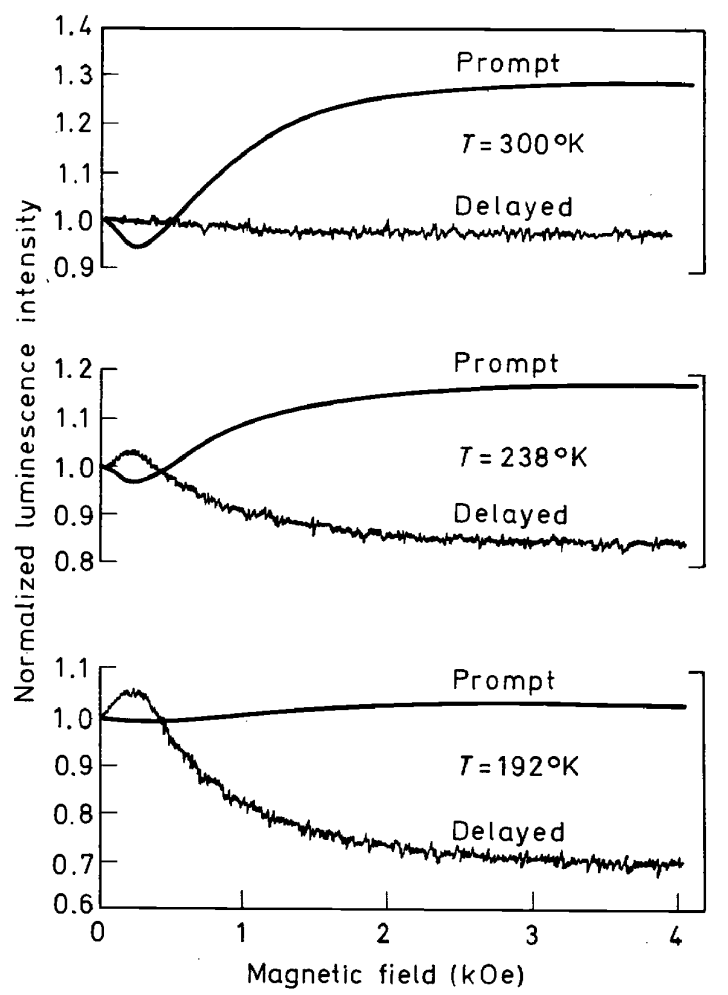

Figure 12. Magnetic field dependence of prompt and delayed fluorescence intensities in a tetracene crystal. The field is oriented at $-20^{\circ}$ with respect to the $b$ axis in the $a b$ plane of the crystal.

(Reproduced by kind permission of the Physical Review. Phys. Rev. B1, 815 (1970))

distinguished. The observed anisotropy is thus a superposition of two sets of level-crossing resonances. Analysis of the angular position of the highfield resonances shows that the tetracene molecule enters the anthracene lattice approximately substitutionally.

\section{Exciton Fission}

Equation (4), the reverse of the triplet fusion reaction, is not observed in the vast majority of molecular crystals since it is too highly endothermic (ca. $4000 \mathrm{~cm}^{-1}$ for anthracene). However, in the case of the tetracene crystal, the $2 T$ level lies only about ${ }^{14} 1200 \mathrm{~cm}^{-1}$ above $S_{1}$ so that fission of a singlet exciton into a pair of triplets is energetically feasible. The occurrence of this process was first suggested by Swenberg and Stacy ${ }^{15}$ to account for the 
anomalously low fluorescence quantum yield of tetracene crystals. The correctness of this suggestion has been verified by experimental demonstration that the prompt fluorescence quantum yield of tetracene is magnetic field dependent ${ }^{16,17}$ (in contrast to fluorescence of other organic crystals) and that this field dependence is the inverse of that of triplet exciton fusion in the same crystal ${ }^{14}$. This is illustrated in Figures 12 and 13.

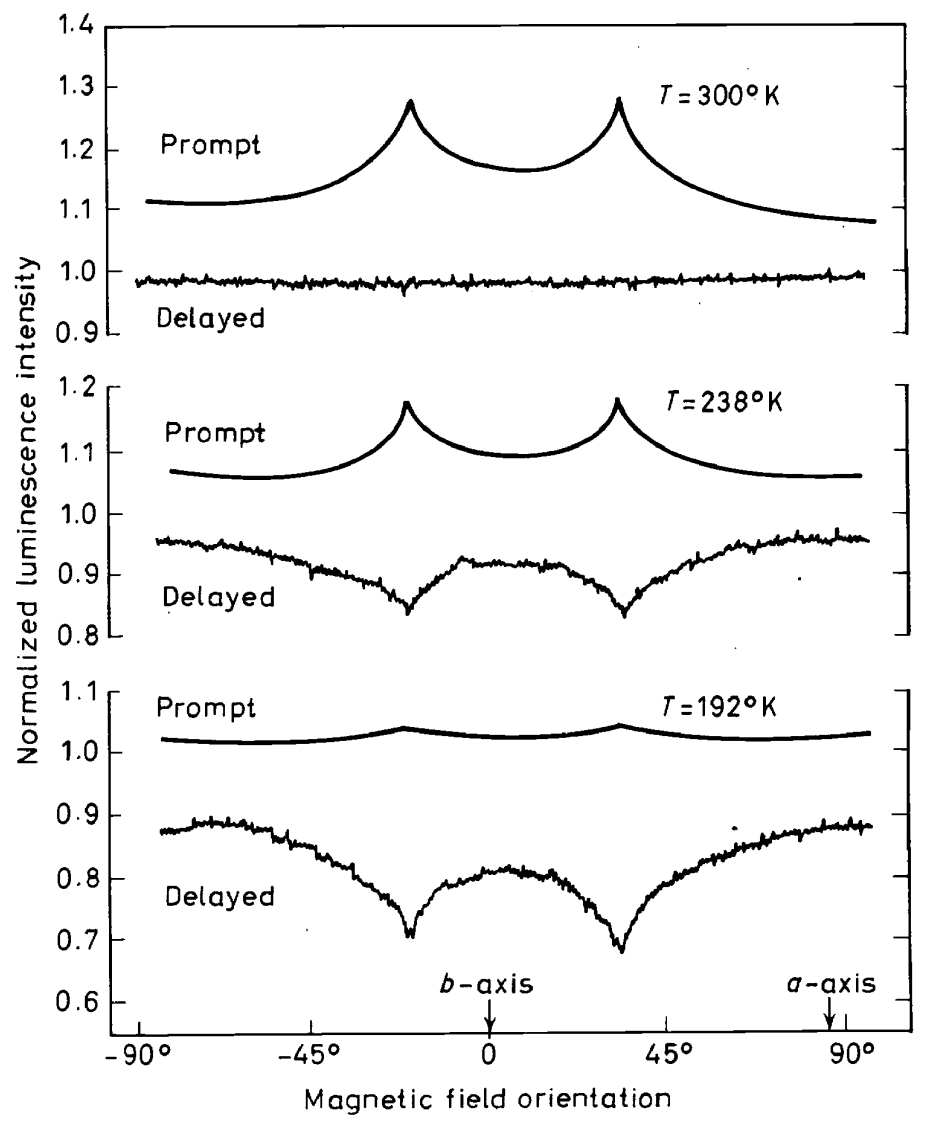

Figure 13. Dependence of prompt and delayed fluorescence intensity on the orientation of a 4 kOe magnetic field in the $a b$ plane of a tetracene crystal. (Reproduced by kind permission of the Physical Review. Phys. Rev. B1, 815 (1970))

At room temperature most singlet excitons produced in the crystal decay via fission, so that the field dependence of the prompt fluorescence intensity is the inverse of the field dependence of the fission rate constant. On the other hand, the delayed fluorescence, in which singlets are being produced by the fusion reaction, shows virtually no field dependence since the singlet production and decay rates have the same field dependence. Since the fission reaction is endothermic, the situation is reversed at low temperatures where most singlets decay radiatively. These effects show clearly in Figures 12 and 13. 


\section{MAGNETIC EFFECTS ON TRIPLET EXCITON INTERACTIONS}

\section{Triplet-Radical Interaction}

It is well known that the lifetimes of triplet excitons are shortened in the presence of paramagnetic impurities in the crystal ${ }^{18}$. Presumably the radicals act as catalysts for intersystem crossing from $T$ to $S_{0}$ by allowing the process of equation (5) to take place without violating spin selection rules. The six spin states of a triplet exciton-doublet radical pair can be analyzed in a manner very similar to that employed for a pair of triplets ${ }^{19}$. The overall spin states will be doublet-quartei mixtures whose field dependence will be

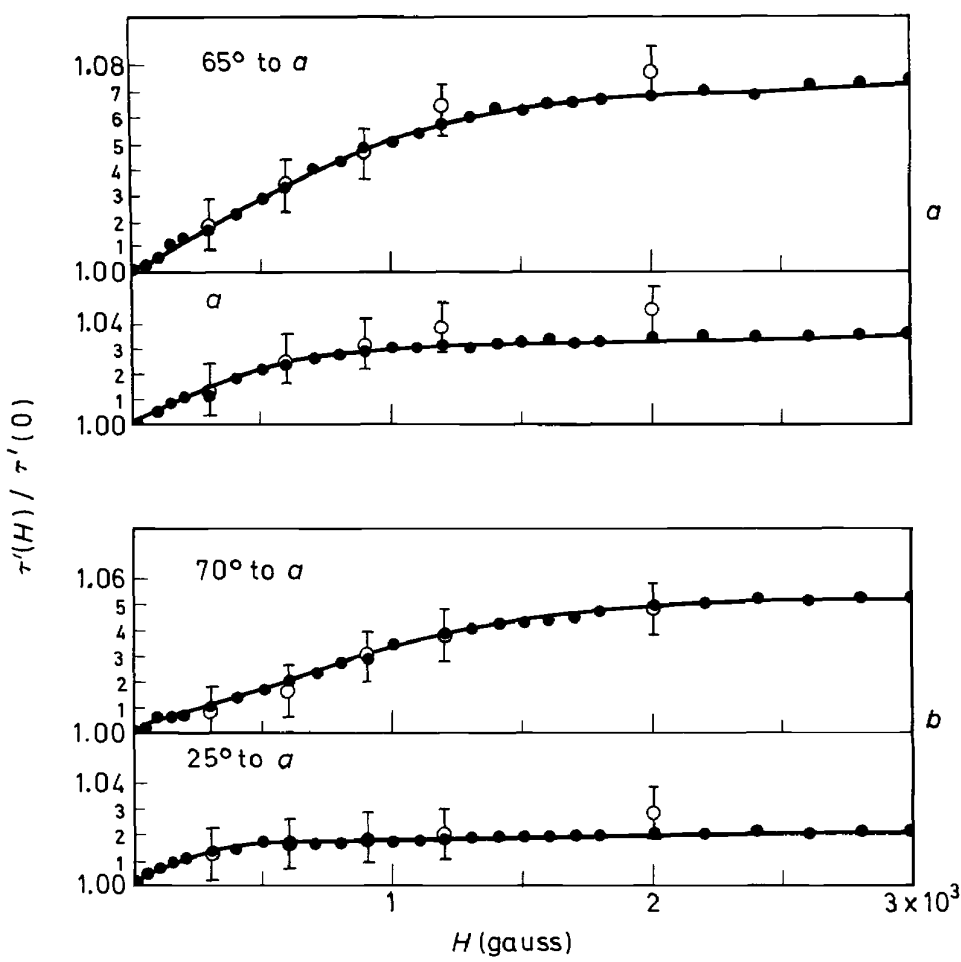

Figure 14. Magnetic field dependence of triplet exciton lifetime in $\mathrm{x}$-irradiated anthracene. (a) dose $4 \times 10^{3} \mathrm{R}, \tau(0)=1.55 \mathrm{msec} . H$ in $a b$ plane in directions at $65^{\circ}$ (resonance) and $0^{\circ}$ with respect to the $a$ axis. (b) dose $1 \times 10^{3} \mathrm{R}, \tau(0)=4.95 \mathrm{msec}$. $H$ in $a c$ plane along $70^{\circ}$ (resonance) and $25^{\circ}$ to $a$ directions. Open circles: direct measurements. Curves drawn from steady-state delayed fluorescence intensity ratios. (Reproduced by kind permission of the Physical Review.

Phys. Rev. Letters 21, 609 (1968))

reflected in a corresponding field dependence of the rate of triplet quenching, which is proportional to the doublet character of the combined spin state since the final state in equation (5) is a pure doublet. In this case the model predicts a monotonic decrease of quenching rate with increasing magnetic field, as well as the existence of a set of high-field level-crossing resonances which will occur at the same positions as the resonances observed in triplettriplet fusion. Experimental results on the quenching of triplet excitons in an anthracene crystal by radicals introduced by $x$-irradiation are shown in Figure $14^{19}$. The qualitative predictions of the model are well confirmed by 
the experimental results, although from these results it is not possible to decide whether the radical simply catalyses intersystem crossing in the host crystal or whether there is energy transfer to the radical.

\section{SUMMARY}

The principal conclusions, which emerge from the work which has been reviewed here, are that the rate of any triplet exciton process which is subject to a spin selection rule is expected to be magnetic field dependent and that analysis of this field dependence can lead to considerable information regarding the details of triplet-exciton interactions. It is perhaps worth emphasizing that the magnetic field effects discussed here do not result from spin polarization or partial alignment of the triplet exciton magnetic moment by the field; the magnetic energies involved in these experiments are entirely negligible compared to thermal energies. Rather, the magnetic field exerts its influence through its effects on the spin wavefunctions of the triplet excitons.

The examples given in the foregoing do not exhaust the potential catalogue of triplet-exciton interactions which could be expected to be affected by a magnetic field. For example, it is known ${ }^{20}$ that triplet excitons interact with free and trapped electrons and holes in organic crystals. Some of the details of this interaction should be amenable to study by magnetic-field effects. Another example is the interaction between triplet states and molecular oxygen $^{21}$. The mechanism by which oxygen quenches triplets could perhaps be clarified by magnetic-field experiments.

\section{REFERENCES}

1 P. Avakian and R. E. Merrifield. Mol. Cryst. 5, 37 (1968).

2 H. Sternlicht and H. M. McConnell. J. Chem. Phys. 35, 1793 (1961).

3 J. H. van der Waals and M. S. de Groot. Mol. Phys. 2, 333 (1959).

4 R. C. Johnson and R. E. Merrifield. Phys. Rev. B1, 896 (1970).

5 R. C. Johnson, R. E. Merrifield, P. Avakian and R. B. Flippen. Phys. Rev. Letters 19, 285 (1967).

${ }^{6}$ R. E. Merrifield. J. Chem. Phys. 48, 4318 (1968);

A. Suna, Phys. Rev. B1, 1716 (1970).

7 R. P. Groff, R. E. Merrifield and P. Avakian. Chem. Phys. Lett. 5, 168 (1970).

${ }^{8}$ D. Haarer, D. Schmid and H. C. Wolf. Phys. Status Solidi 23, 633 (1967).

9 C. A. Parker. Photoluminescene of Solutions. Elsevier Publishing Company, Amsterdam (1968).

10 L. R. Faulkner and A. F. Bard. J. Am. Chem. Soc. 91, 6495 (1969).

11 P. Avakian; R. P. Groff, R. E. Kellogg, R. E. Merrifield and A. Suna. Proceedings of the International Conference on Organic Scintillators and Liquid Scintillation Counting, San Franciso, July 1970 (Academic Press, to be published).

12 R. P. Groff, R. E. Merrifield, P. Avakian and Y. Tomkiewicz. Phys. Rev. Letters 25, 105 (1970).

13 A. M. Mathieson, J. M. Robertson and V. C. Sinclair. Acta Cryst. 3, 245 (1950).

14 R. P. Groff, P. Avakian and R. E. Merrifield. Phys. Rev. B1, 815 (1970).

15 C. E. Swenberg and W. T. Stacey. Chem. Phys. Lett. 2, 329 (1968).

16 N. Geacintov, M. Pope and F. Vogel. Phys. Rev. Letters 22, 593 (1969).

17 R. E. Merrifield, P. Avakian and R. P. Groff. Chem. Phys. Lett. 3, 155 (1969).

18 S. Z. Weisz, P. Richardson, A. Cobas and R. C. Jarnagin. Mol. Cryst. 3, 168 (1967).

19 V. Ern and R. E. Merrifield. Phys. Rev. Letters 21, 609 (1968).

20 W. Helfrich. Phys. Rev. Letters 16, 401 (1966).

${ }^{21}$ S. P. McGlynn, T. Azumi and M. Kinoshita. Molecular Spectroscopy of the Triplet State (Prentice-Hall, Englewood Cliffs, New Jersey, 1969), pp. 284-293. 